\title{
Clostridium difficile in Inflammatory Bowel Disease: A Retrospective Study
}

\author{
William Gillespie, ${ }^{1}$ Neil Marya, ${ }^{2}$ Julien Fahed, ${ }^{3}$ Gregory Leslie, ${ }^{4}$ Krunal Patel, ${ }^{5}$ and \\ David R. Cave \\ ${ }^{1}$ Department of Medicine, University of Massachusetts School of Medicine, 55 Lake Avenue North, Worcester, MA 01604, USA \\ ${ }^{2}$ Department of Gastroenterology, University of California Los Angeles, Los Angeles, CA, USA \\ ${ }^{3}$ Department of Gastroenterology, Aurora Healthcare, Milwaukee, WI, USA \\ ${ }^{4}$ Department of Medicine, University of Massachusetts, Worcester, MA, USA \\ ${ }^{5}$ Department of Gastroenterology, University of Massachusetts, Worcester, MA, USA
}

Correspondence should be addressed to William Gillespie; william.gillespie@umassmemorial.org

Received 6 June 2017; Accepted 24 July 2017; Published 4 October 2017

Academic Editor: Paolo Gionchetti

Copyright (C) 2017 William Gillespie et al. This is an open access article distributed under the Creative Commons Attribution License, which permits unrestricted use, distribution, and reproduction in any medium, provided the original work is properly cited.

\begin{abstract}
Aim. To investigate the epidemiology and risk factors of Clostridium difficile infections (CDI) in patients with inflammatory bowel disease (IBD). Methods. This is a retrospective study of patients diagnosed with IBD. 1006 charts were screened and 654 patients met the inclusion criteria. Patients were divided into 2 cohorts based on the presence of prior diagnosis of CDI. Statistical analysis with Pearson's chi-squared and two-sample t-test was performed. Results. The incidence of CDI among IBD patients was $6.7 \%$. There was equal prevalence of CDI among Crohn's disease (CD) $(n=21,49 \%)$ and ulcerative colitis (UC) $(n=22,51 \%)$. IBD patients acquired CDI at a mean age of 42.7 years, with $56 \%$ of infections acquired in the community and only $28 \%$ associated with healthcare. Only $30 \%$ of IBD patients with CDI had prior antibiotic use, and $16 \%$ had prior steroid use. IBD patients were significantly more likely to require biologic therapy ( $57 \%$ versus $37 \%, p<0.01)$ and have extraintestinal manifestations of IBD $(43 \%$ versus $28 \%, p<0.02)$. Conclusions. IBD patients are more susceptible to CDI at a younger age and often lack traditional risk factors. IBD patients with at least one CDI were more likely to require biologic therapy and had greater rates of extraintestinal manifestations.
\end{abstract}

\section{Introduction}

Clostridium difficile is the major cause of infectious diarrhea in hospitalized patients [1] and is the primary infectious cause of pseudomembranous colitis [2]. Recent studies have shown that infections with Clostridium difficile (CDI) have shown increased incidence, severity, and recurrence rates over the past 10 years [3]. In addition, such infection has recently surpassed that of methicillin-resistant Staphylococcus aureus as the leading hospital-acquired infection in the United States $[4,5]$. These infections lead to longer hospital stays, more frequent readmissions, and increased mortality among hospitalized patients [4]. Healthcare costs related to CDI are estimated to be around $\$ 1.8$ billion annually in the United States, can add over $\$ 11,000$ to a patient's hospital stay, and increase the length of stay by an average of 3.3 days [6]. There are several risk factors for CDI, with antibiotic use being the most well-known. Others include advanced age, increased severity of underlying illness, prior hospitalization, feeding tube use, gastrointestinal surgery, and potentially proton-pump inhibitors [7].

Patients with inflammatory bowel disease (IBD), which includes Crohn's disease (CD) and ulcerative colitis (UC), have been shown to have increased CDI rates and disproportionately higher morbidity and mortality compared to CDI patients without IBD $[8,9]$. Classical risk factors may not be found in many IBD patients, and clinical findings such as pseudomembranes may not be present [3]. In contrast to archetypal epidemiological studies, CDI is now being increasingly recognized as a cause of diarrhea in the community, 
especially in younger individuals and in populations lacking the traditional risk factors [10-12]. This study seeks to further characterize the epidemiology and risk factors for $C$. difficile infections in IBD patients.

\section{Methods}

This was a retrospective study. The medical records of 1006 patients who carried the ICD 9 code for IBD (ICD-9: 555.x and 556.x) at a single large academic medical center from October 1, 2014, to September 30, 2015, were reviewed. Approval was obtained from the Committee for the Protection of Human Subjects for the finalized study protocol. Eligible patients were identified through a search of the electronic medical record, and data was deidentified and randomized prior to analysis. Eligible patients had a previous diagnosis of IBD based on ICD coding along with appropriate clinical symptoms and compatible endoscopic or imaging findings. 352 patients were excluded due to insufficient data to confirm IBD diagnosis or inappropriate coding. For the remaining 654 patients, extensive background epidemiologic and comorbid data were collected for each subject and incorporated into the final analysis. The data extracted from the charts included the current age of the patients, the age of IBD diagnosis, the type of IBD, peak therapy between the time of diagnosis and the current time of the study, extraintestinal manifestations, hospitalization data, inflammatory markers, and antibiotic and steroid use, among many others (Tables 1-3). For subanalysis, patients were divided into those with prior documented CDI and those without documented CDI. Statistical analysis with Pearson's chi-squared test and two-sample $t$-test was performed.

\section{Results}

3.1. Epidemiology. A total of 654 IBD patients were studied. 43 patients $(6.7 \%)$ had at least one confirmed CDI. A review of baseline characteristics revealed a mean patient age of 42.7 years at the time of the first CDI, with $55.8 \%$ male predominance (Tables 1 and 3). This gender distribution is similar to that of IBD patients without prior CDI $(52.5 \%$ male). Patients with prior CDI were more likely to have been hospitalized with at least one IBD-related admission compared to IBD patients without CDI (72\% versus $42 \%$, $p<0.001)$. The mean number of IBD-related hospitalizations of patients with CDI was 2.9, and that of IBD patients without CDI was $1.12(p<0.001) .43 \%$ of IBD patients with past CDI eventually required surgery, while only $33.5 \%$ of those without prior CDI ultimately required a surgical procedure (but this was not statistically significant with $p=0.15)$.

3.2. CDI Characteristics. The mean age of diagnosis of CDI in IBD patients was 42.7 years (Table 3 ). Interestingly, $56 \%$ of these patients had community-acquired CDI (as per the most recent IDSA guidelines [13]), with only $28 \%$ of CDI being hospital-acquired and $16 \%$ being indeterminate. Of those patients with CDI, only $30 \%$ used an antibiotic
TABLE 1: IBD demographics.

\begin{tabular}{lcc}
\hline & $\begin{array}{c}\text { IBD patients with } \\
\text { prior CDI }(n=43)\end{array}$ & $\begin{array}{c}\text { IBD patients without } \\
\text { prior CDI }(n=611)\end{array}$ \\
\hline Male, $n(\%)$ & $24(55.8)$ & $321(52.5)$ \\
Crohn's disease, $n(\%)$ & $21(48.8)$ & $344(56.3)$ \\
Ileal & $5(23.8)$ & $89(25.6)$ \\
Colonic & $5(23.8)$ & $81(23.2)$ \\
Ileocolonic & $10(47.6)$ & $150(43.1)$ \\
Isolated upper & $1(4.8)$ & $24(6.9)$ \\
disease & $22(51.2)$ & $267(43.6)$ \\
Ulcerative colitis, $n(\%)$ & $10(45.5)$ & $119(46.3)$ \\
Pancolitis & $7(31.8)$ & $60(23.3)$ \\
Left-sided colitis & $2(9)$ & $50(19.4)$ \\
Proctosigmoiditis & $3(13.6)$ & $28(10.9)$ \\
Proctitis &
\end{tabular}

within 1 month of diagnosis. Additionally, only $16 \%$ used steroids within 1 month prior to CDI diagnosis. For the initial treatment of CDI, 44\% received oral metronidazole, $32.5 \%$ received oral vancomycin, and $11 \%$ received intravenous solumedrol. Seven patients (16\%) had more than one CDI (occurring $>8$ weeks after completion of treatment and resolution of symptoms), and two patients had multiple recurrences. Of the patients with recurrent disease, all had initial treatment with PO metronidazole. The average time to disease clearance (defined as resolution of symptoms after completion of treatment or documented negative PCR) was 20.1 days.

3.3. IBD Characteristics. IBD patients with at least one CDI were significantly more likely to require escalation to biologic therapy when compared to IBD patients without prior CDI ( $57 \%$ versus $38 \%, p<0.01$, Table 2 ). Only 14 patients were actively on a biologic at the time of their first CDI diagnosis. Of a total of 256 patients on biologic agents, only 14 patients (5.5\% of all biologic users) developed CDI while on a biologic. There were no significant differences among the types of biologic. Only $18 \%$ of patients with prior CDI were able to control their IBD with salicylates alone, while $31 \%$ of those without prior CDI attained adequate control with salicylates, but this was not statistically significantly different $(p=0.08)$.

IBD patients with prior CDI had a higher rate of extraintestinal manifestations of IBD than those without prior CDI ( $43 \%$ versus $28 \%, p=0.02)$. These included inflammatory arthritis, osteoporosis, osteomalacia, pyoderma gangrenosum, psoriasis, and chronic pancreatitis. The lowest mean albumin of those with prior CDI was 2.98, compared to 3.45 in those without prior CDI $(p<0.001)$. Mean peak ESR and CRP were also more elevated in those with CDI, but this was not statistically significant $(p=0.07$ and $p=0.06$, resp.).

With regard to the IBD subtype in patients with prior CDI, 51\% had UC and 49\% had CD. This distribution was similar to that of IBD patients without CDI (43\% UC and 
TABLE 2: IBD characteristics.

\begin{tabular}{lccc}
\hline & $\begin{array}{c}\text { IBD patients with prior } \\
\text { CDI }(n=43)\end{array}$ & $\begin{array}{c}\text { IBD patients without prior } \\
\text { CDI }(n=611)\end{array}$ & $\begin{array}{c}\text { Univariate analysis } \\
p \text { value }\end{array}$ \\
\hline$\geq 1$ IBD-related hospitalization, $n(\%)$ & $32(72)$ & $255(41.7)$ & $p<0.001$ \\
Number of IBD-related hospitalizations, mean \pm SD & $2.9 \pm 3.1$ & $1.12 \pm 2.27$ & $p<0.001$ \\
Surgery for IBD, $n$ (\%) & $19(43.2)$ & $205(33.5)$ & $p=0.150$ \\
Biologic as peak IBD therapy, $n$ (\%) & $25(56.8)$ & $231(37.8)$ & $p=0.008$ \\
Salicylate as peak IBD therapy, $n(\%)$ & $8(18)$ & $189(30.9)$ & $p=0.088$ \\
Extraintestinal manifestations of IBD, $n(\%)$ & $19(43)$ & $174(28.4)$ & $p=0.021$ \\
Lowest albumin, mean \pm SD & $2.98 \pm 0.83$ & $3.45 \pm 0.79$ & $p<0.001$ \\
Peak CRP, mean \pm SD & $55.9 \pm 64.8$ & $37.8 \pm 58.3$ & $p=0.065$ \\
Peak ESR, mean \pm SD & $43.5 \pm 31.5$ & $35 \pm 28.2$ & $p=0.071$ \\
\hline
\end{tabular}

$\mathrm{CRP}=\mathrm{C}$-reactive protein, $\mathrm{ESR}=$ erythrocyte sedimentation rate.

TABLE 3: CDI characteristics.

\begin{tabular}{lc}
\hline & $\begin{array}{c}\text { IBD patients with prior } \\
\text { CDI }(n=43)\end{array}$ \\
\hline $\begin{array}{l}\text { Age at first CDI (years), mean } \pm \text { SD } \\
\text { CDI etiology, } n(\%)\end{array}$ & $42.7 \pm 19.9$ \\
Community-acquired & $24(56)$ \\
Hospital-acquired & $12(28)$ \\
Indeterminate & $7(16)$ \\
Antibiotic use prior to CDI, $n(\%)$ & $13(30)$ \\
Steroid use prior to CDI, $n(\%)$ & $7(16.3)$ \\
Biologic use at the time of CDI, $n(\%)$ & $14(32.5)$ \\
Time to CDI clearance (days), mean \pm SD & $20.6 \pm 14.27$ \\
\hline
\end{tabular}

$57 \%$ CD). These results show no statistically significant correlation between the IBD subtype and CDI. Overall, $8.3 \%$ of all UC patients and $6 \%$ of all CD patients studied had at least one CDI. As expected, the anatomical distribution of IBD in those with CDI predominantly involved the colon. Of those with CDI in UC, $45 \%$ had pancolitis, $31.8 \%$ had left-sided colitis, 9\% had proctosigmoiditis, and 13\% had proctitis. These results very closely mirror the distribution of UC in those without CDI. Similarly, in patients with CDI in CD, 23\% had either isolated ileal or colonic disease, $48 \%$ had ileocolonic disease, and only $4 \%$ had isolated upper disease. This distribution is almost identical to the prevalence of $\mathrm{CD}$ subtypes in patients without CDI.

\section{Discussion}

Our study analyzed a large $(n=654)$ cohort of IBD patients for epidemiologic and comorbid conditions. Of these IBD patients, $43(6.7 \%)$ had proven CDI. This incidence is concordant with that of other epidemiological studies of CDI in IBD, with rates ranging from $3 \%$ to $6 \%[3,14,15]$. This rate has nearly doubled over the past decade and is far greater than that in those patients without IBD $(0.04 \%$ to $0.08 \%)$ [14]. The majority of CDI in this study were diagnosed using PCR for the $C$. difficile toxin B gene in patients with clinical symptoms of infection. Two patients were diagnosed with CDI using an immunoassay for toxins A and B.

The mean age for patients with IBD who contracted CDI was only 42.7 years in this study. This is in stark contrast to the mean age of non-IBD patients most susceptible to CDI, which ranges from 65 to 75 years of age depending on the study [16-20]. Increased age is a well-recognized risk factor for CDI; however, some studies have shown that IBD patients appear to be more susceptible at a younger age $[12,15]$. It is tempting to attribute this skewed age distribution to increased hospitalizations, antibiotic use, and/or immunosuppression in younger IBD patients, but these factors do not account for the majority of CDI cases in this study.

Our data shows that those IBD patients with prior CDI were significantly more likely to have had at least one hospitalization for an IBD-related cause (72\% versus $41 \%)$. Additionally, those IBD patients with prior CDI were significantly more likely to have multiple hospitalizations (mean 2.9 versus 1.1). Despite the increased incidence and number of hospitalizations in those with prior CDI, our data shows that $56 \%$ of infections were community-acquired. This is in contrast to the data from Europe, Canada, and the United States suggesting a rate of $20-27 \%$ communityacquired CDI in the general population [21-23].

In this study, we used the most recent Infectious Diseases Society of America recommendations, which state that CDI is community-acquired if the patient has not been discharged from a healthcare facility in the previous 12 weeks and symptoms begin in the community or within 48 hours of admission to a hospital. Hospital-acquired CDI is defined as a symptom onset more than $48 \mathrm{hrs}$ after admission or less than 4 weeks after discharge, and CDI is considered indeterminate if symptoms occur in the community between 4 and 12 weeks after discharge [14]. In contrast to the $56 \%$ rate of community-acquired CDI, only $28 \%$ of infections were hospital-acquired and $16 \%$ indeterminate. This is directly a counter to the traditional view of CDI as a predominantly healthcare-associated infection. Our results are consistent with those of other retrospective population studies of both adults and children showing that IBD patients with CDI were younger and more often had acquired infections as 
outpatients compared with patients without IBD $[15,24]$. The majority of CDI were community-acquired; only $30 \%$ had antibiotic use within one month of diagnosis. These findings demonstrate the lack of traditional risk factors for CDI in patients with IBD. This awareness is crucial for accurate diagnosis. CDI may mimic or precipitate an IBD flare, but these two entities have distinctly divergent treatment plans. Empiric treatment with glucocorticoids in CDI without appropriate antibiotic coverage has been associated with higher short-term mortality [25], so proper diagnosis is essential.

In regard to immunosuppression, those IBD patients with at least one CDI were significantly more likely to require escalation to biologic therapy when compared to IBD patients without CDI (57\% versus 38\%). The overwhelming majority of biologic agents observed in this study were the TNF-alpha inhibitors infliximab and adalimumab. Similarly, only $18 \%$ of patients with prior CDI were able to control their disease with salicylates alone, while $31 \%$ of those without CDI attained adequate control with salicylates (but this was not statistically significant with $p=0.08$ ). These findings could represent an increased susceptibility to CDI in those patients with more severe or aggressive IBD. In IBD, dysfunction of regulatory $\mathrm{T}$ cells or antigen-presenting cells can lead to a loss of tolerance to ubiquitous microbial antigens [26]. The concentrations of mucosal bacteria of the colon have been shown to increase progressively with the severity of disease, both in the inflamed and noninflamed colon [27]. While the results of specific microbial dysbiosis in IBD vary between studies, the most consistent observation is reduced bacterial diversity [28]. It is known that reduced microbial diversity in the gut is a common pathogenic pathway for CDI; therefore, those patients with more severe IBD may be more innately susceptible to CDI.

Perhaps, a more apparent cause of increased CDI in this population would be the direct immunosuppressive effects of biologic agents themselves. However, studies have shown conflicting data on the role of TNF-alpha inhibitors such as infliximab and adalimumab in increasing susceptibility to CDI [28-30]. In our study, the majority of patients who had CDI would eventually require a biologic; however, only 14 patients were actively on a biologic at the time of CDI diagnosis. In addition, there was no recurrence of CDI after patients were started on a biologic. Overall, only $5.4 \%$ of the 256 patients on a biologic agent were diagnosed with CDI. Similarly, only $16 \%$ of patients were on systemic corticosteroids or immunomodulators within one month of CDI diagnosis. While immunosuppressive agents certainly increase susceptibility to opportunistic infections, they did not appear to be the principal risk factor for CDI in this study.

Patients with at least one CDI were more likely to have extraintestinal manifestations of IBD than those patients without CDI (43\% versus $28 \%$ ). These included inflammatory arthritis, osteoporosis, osteomalacia, pyoderma gangrenosum, psoriasis, and chronic pancreatitis. While both arthritis and osteoporosis are very common conditions, the majority of these patients developed these conditions at an age younger than 60 years. IBD patients with at least one
CDI also had lower mean albumin than those without CDI (2.98 versus 3.45 ).

There are some limitations to this study. This study was retrospective. We were unable to evaluate the severity of CDI due to the large proportion of community-acquired and indeterminate infections. Data was provided by a large number of outside providers with variability in data collected or accessible around the time of diagnosis. Similarly, the CDI cohort consists of only those patients who were admitted to our hospital with CDI or were followed up by one of our outpatient providers around the time of diagnosis. There are likely patients in the IBD cohort who were diagnosed or treated for CDI at outside facilities without our knowledge, leading to underestimation of CDI incidence and an incomplete CDI cohort. Finally, the toxin B PCR cannot distinguish between new infection and asymptomatic carriage, complicating diagnosis in this population given the similarity of symptoms between CDI and an IBD flare.

This study demonstrates an equal prevalence of CDI in UC and CD. It shows that IBD patients are more susceptible to CDI at a younger age and often lack traditional risk factors such as recent hospitalization or antibiotic use. Additionally, IBD patients with at least one CDI were more likely to require biologic therapy, had lower success with salicylates, had lower mean albumin, and had greater rates of extraintestinal manifestations.

\section{Disclosure}

An abstract based on initial data from this study database was presented at the annual conference for the American College of Gastroenterology in Honolulu, October 2015. The following people made contributions to that abstract: Ackerman, Rachel, MD (Department of Medicine, Tufts Medical Center)_data collection; Hoffman, Benjamin (Isenberg School of Management)—data collection; Foley, Anne, BA (Department of Gastroenterology, University of Massachusetts)data collection; Hyatt, Benjamin, MD (Department of Gastroenterology, University of Massachusetts)_study design; and Pellish, Randall, MD (Department of Gastroenterology, University of Massachusetts)—study design.

\section{Conflicts of Interest}

No authors have conflicts of interest to report.

\section{References}

[1] S. S. Magill, J. R. Edwards, W. Bamberg et al., "Multistate point-prevalence survey of health care associated infections," The New England Journal of Medicine, vol. 370, pp. 11981208, 2014.

[2] J. G. Bartlett, T. W. Chang, M. Gurwith, S. L. Gorbach, and A. B. Onderdonk, "Antibiotic-associated pseudomembranous colitis due to toxin-producing clostridia," The New England Journal of Medicine, vol. 298, no. 10, pp. 531-534, 1978.

[3] J. F. Rodemann, E. R. Dubberke, K. A. Reske, D. H. Seo, and C. D. Stone, "Incidence of Clostridium difficile infection in inflammatory bowel disease," Clinical Gastroenterology and Hepatology, vol. 5, no. 3, pp. 339-344, 2007. 
[4] F. C. Lessa, Y. Mu, W. M. Bamberg et al., "Burden of Clostridium difficile infection in the United States," The New England Journal of Medicine, vol. 372, no. 9, pp. 825-834, 2015.

[5] B. A. Miller, L. F. Chen, D. J. Sexton, and D. J. Anderson, "Comparison of the burdens of hospital-onset, healthcare facility-associated Clostridium difficile infection and of healthcare-associated infection due to methicillin-resistant Staphylococcus aureus in community hospitals," Infection Control and Hospital Epidemiology, vol. 32, no. 4, pp. 387390, 2011.

[6] E. Zimlichman, D. Henderson, O. Tamir et al., "Health careassociated infections: a meta-analysis of costs and financial impact on the US health care system," JAMA Internal Medicine, vol. 173, no. 22, pp. 2039-2046, 2013.

[7] G. E. Bignardi, "Risk factors for Clostridium difficile infection," The Journal of Hospital Infection, vol. 40, no. 1, pp. 1-15, 1998.

[8] S. Khanna and D. S. Pardi, "IBD: poor outcomes after Clostridium difficile infection in IBD," Nature Reviews Gastroenterology \& Hepatology, vol. 9, no. 6, pp. 307-308, 2012.

[9] A. N. Ananthakrishnan, E. L. McGinley, and D. G. Binion, "Excess hospitalisation burden associated with Clostridium difficile in patients with inflammatory bowel disease," Gut, vol. 57, no. 2, pp. 205-210, 2008.

[10] Centers for Disease Control and Prevention (CDC), "Severe Clostridium difficile-associated disease in populations previously at low risk - four states, 2005," MMWR Morbidity and Mortality Weekly Report, vol. 54, no. 47, pp. 1201-1205, 2005.

[11] S. Khanna and D. S. Pardi, "The growing incidence and severity of Clostridium difficile infection in inpatient and outpatient settings," Expert Review of Gastroenterology \& Hepatology, vol. 4, no. 4, pp. 409-416, 2010.

[12] S. Khanna, D. S. Pardi, S. L. Aronson et al., "The epidemiology of community-acquired Clostridium difficile infection: a population-based study," The American Journal of Gastroenterology, vol. 107, no. 1, pp. 89-95, 2012.

[13] S. H. Cohen, D. N. Gerding, S. Johnson et al., "Clinical practice guidelines for Clostridium difficile infection in adults: 2010 update by the Society for Healthcare Epidemiology of America (SHEA) and the Infectious Diseases Society of America (IDSA)," Infection Control and Hospital Epidemiology, vol. 31, no. 5, pp. 431-455, 2010.

[14] G. C. Nguyen, G. G. Kaplan, M. L. Harris, and S. R. Brant, “A national survey of the prevalence and impact of Clostridium difficile infection among hospitalized inflammatory bowel disease patients," The American Journal of Gastroenterology, vol. 103, no. 6, pp. 1443-1450, 2008.

[15] M. Issa, A. Vijayapal, M. B. Graham et al., "Impact of Clostridium difficile on inflammatory bowel disease," Clinical Gastroenterology and Hepatology, vol. 5, no. 3, pp. 345-351, 2007.

[16] P. Soler, F. Nogareda, and R. Cano, "Rates of Clostridium difficile infection in patients discharged from Spanish hospitals, 1997-2005," Infection Control and Hospital Epidemiology, vol. 29, no. 9, pp. 887-889, 2008.

[17] F. Burckhardt, A. Friedrich, D. Beier, and T. Eckmanns, "Clostridium difficile surveillance trends, Saxony, Germany," Emerging Infectious Diseases, vol. 14, pp. 691-692, 2008.

[18] J. Lucado, C. Gould, and A. Elixhauser, "Clostridium difficile infections (CDI) in hospital stays, 2009: statistical brief \#124," in Healthcare Cost and Utilization Project (HCUP) Statistical Briefs, Agency for Healthcare Research and Quality (US), Rockville (MD), 2006.
[19] K. R. Reveles, G. C. Lee, N. K. Boyd, and C. R. Frei, "The rise in Clostridium difficile infection incidence among hospitalized adults in the United States: 2001-2010," American Journal of Infection Control, vol. 42, no. 10, pp. 1028-1032, 2014.

[20] M. H. Wilcox, N. Shetty, W. N. Fawley et al., "Changing epidemiology of Clostridium difficile infection following the introduction of a national ribotyping-based surveillance scheme in England," Clinical Infectious Diseases, vol. 55, no. 8, pp. 1056-1063, 2012.

[21] M. H. Wilcox, L. Mooney, R. Bendall, C. D. Settle, and W. N. Fawley, "A case-control study of community-associated Clostridium difficile infection," Journal of Antimicrobial Chemotherapy, vol. 62, no. 2, pp. 388-396, 2008.

[22] P. K. Kutty, C. W. Woods, A. C. Sena et al., "Risk factors for and estimated incidence of community-associated Clostridium difficile infection, North Carolina, USA," Emerging Infectious Diseases, vol. 16, pp. 197-204, 2010.

[23] P. J. Lambert, M. Dyck, L. H. Thompson, and G. W. Hammond, "Population-based surveillance of Clostridium difficile infection in Manitoba, Canada, by using interim surveillance definitions," Infection Control and Hospital Epidemiology, vol. 30, no. 10, pp. 945-951, 2009.

[24] M. Issa, A. N. Ananthakrishnan, and D. G. Binion, "Clostridium difficile and inflammatory bowel disease," Inflammatory Bowel Diseases, vol. 14, no. 10, pp. 1432-1442, 2008.

[25] R. Das, P. Feuerstadt, and L. J. Brandt, "Glucocorticoids are associated with increased risk of short-term mortality in hospitalized patients with clostridium difficile-associated disease," The American Journal of Gastroenterology, vol. 105, no. 9, pp. 2040-2049, 2010.

[26] S. Khanna and P. K. Tosh, “A clinician's primer on the role of the microbiome in human health and disease," Mayo Clinic Proceedings, vol. 89, no. 1, pp. 107-114, 2014.

[27] K. Matsuoka and T. Kanai, "The gut microbiota and inflammatory bowel disease," Seminars in Immunopathology, vol. 37, no. 1, pp. 47-55, 2015.

[28] S. Schneeweiss, J. Korzenik, D. H. Solomon, C. Canning, J. Lee, and B. Bressler, "Infliximab and other immunomodulating drugs in patients with inflammatory bowel disease and the risk of serious bacterial infections," Alimentary Pharmacology \& Therapeutics, vol. 30, no. 3, pp. 253-264, 2009.

[29] R. Kariv, U. Navaneethan, P. G. Venkatesh, R. Lopez, and B. Shen, "Impact of Clostridium difficile infection in patients with ulcerative colitis," Journal of Crohn's \& Colitis, vol. 5, no. 1, pp. 34-40, 2011.

[30] M. A. Engel and M. F. Neurath, "New pathophysiological insights and modern treatment of IBD," Journal of Gastroenterology, vol. 45, no. 6, pp. 571-583, 2010. 


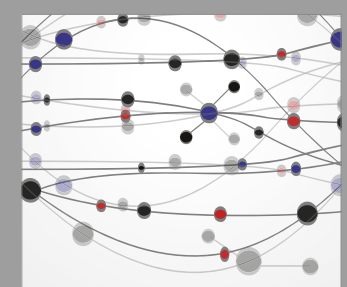

The Scientific World Journal
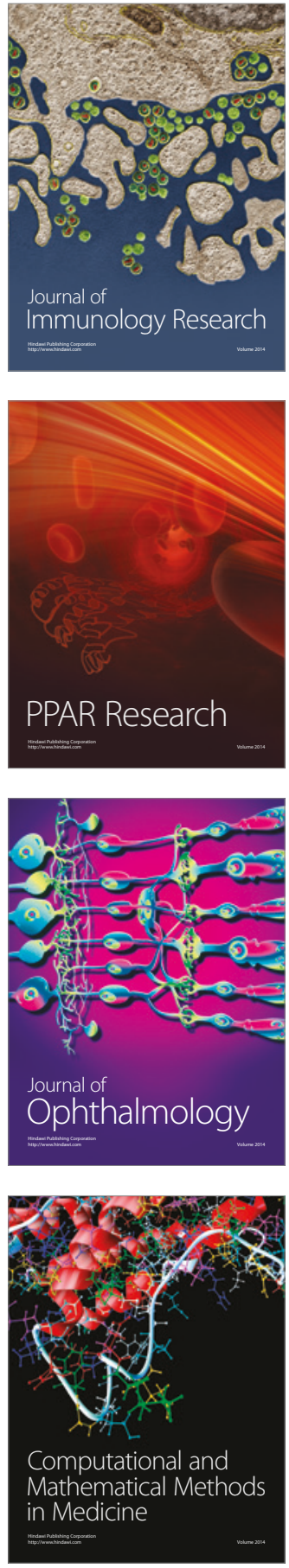

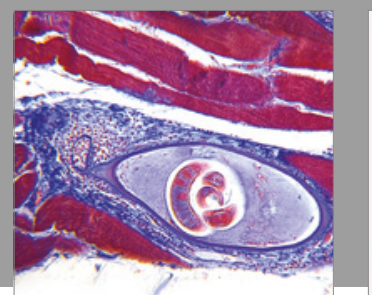

Gastroenterology Research and Practice
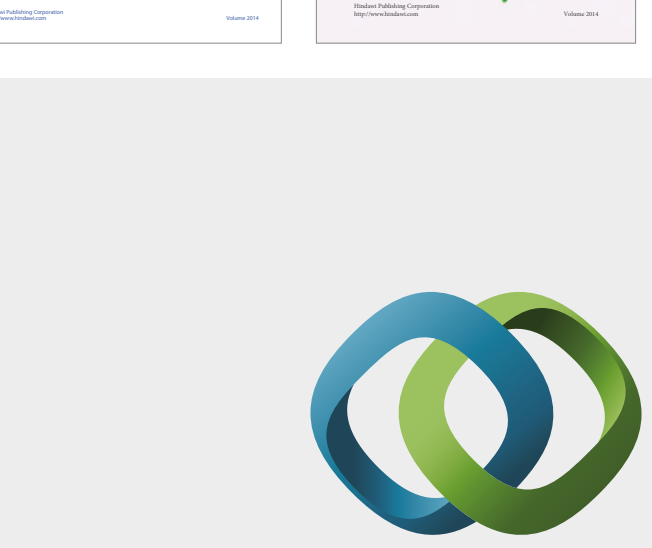

\section{Hindawi}

Submit your manuscripts at

https://www.hindawi.com
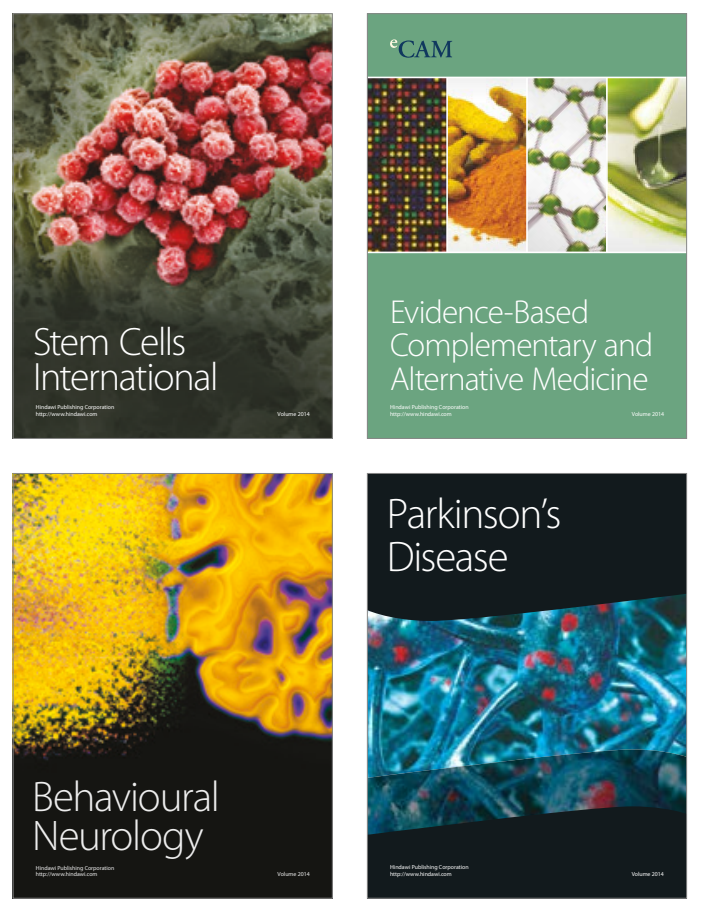
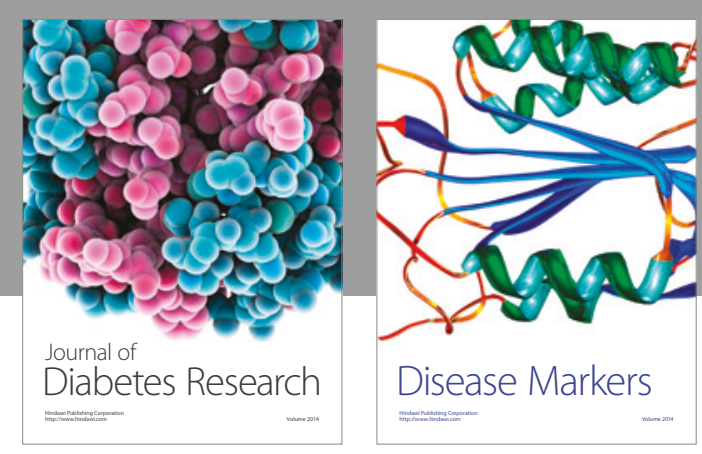

Disease Markers
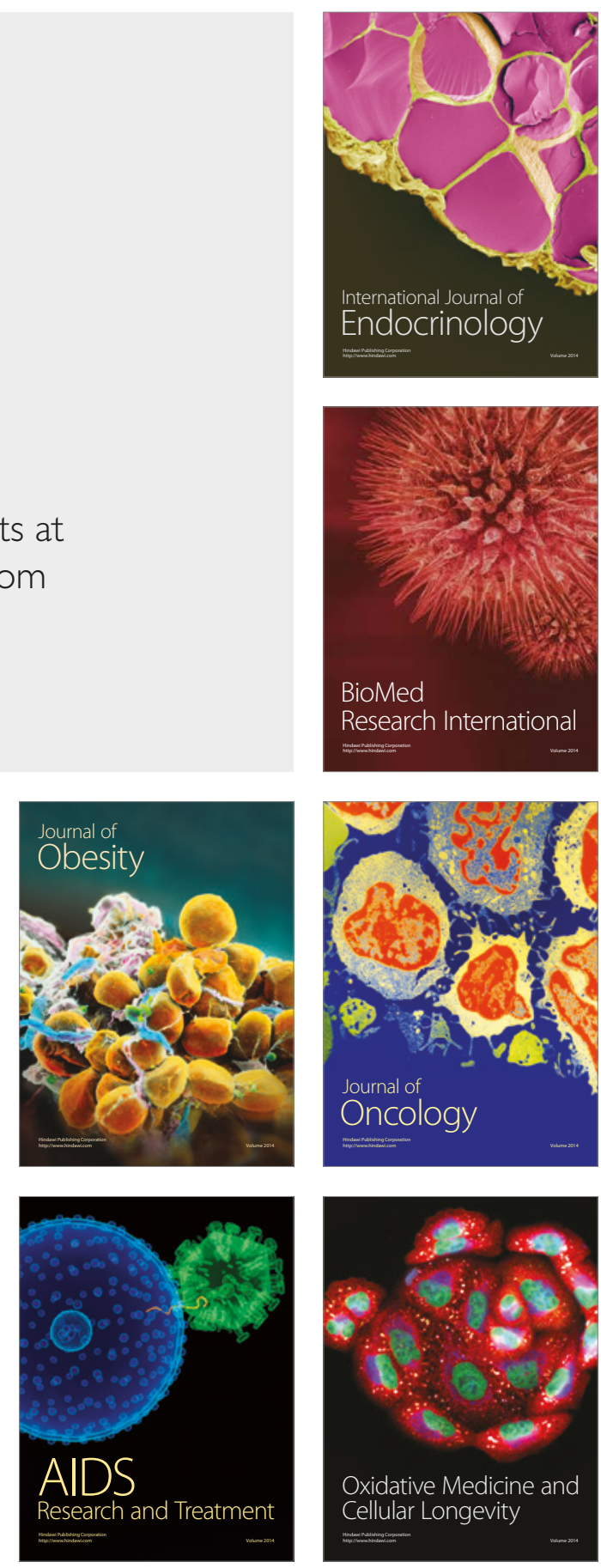